\title{
Topical issue Frontiers of ion trap and atomic physics: Wolfgang Paul 100
}

\author{
Günter Werth · Ferdinand Schmidt-Kaler • \\ Rainer Blatt
}

(C) Springer-Verlag Berlin Heidelberg 2014

When Wolfgang Paul and colleagues from the University of Bonn published their paper "Ein Ionenkäfig" in 1958, it was not at all obvious what kind of applications might result. For the next 15 years, aside from mass analysis in chemistry, the publications involving ion trap physics were very few. The situation changed dramatically when lasers became available as a reliable instrument for spectroscopy of atomic ions. The combination of an ion trap and a tunable laser turned out to be extremely fruitful, and a new field of physics emerged from this combination. Ion traps with their long storage, observation and coherence time allowed for the realization of laser cooling in 1978, and the preparation and spectacular observation of a single trapped ion. Soon it became obvious that the benign environment of ion traps, the well-controlled electric and magnetic fields, allowed for the development of novel hyperfine or optical frequency standards. The Nobel Prize in Physics 1989 was awarded to Wolfgang Paul and Hans Dehmelt "for the development of the ion trap technique," which has led to a dramatic development of atomic precision spectroscopy in the 1980s. The Nobel Committee acknowledged that "the techniques have reached an unprecedented level of precision, and the development does not yet seem to have culminated." As predicted, the field has seen in the

G. Werth $(\bowtie)$

Institut für Physik, Johannes Gutenberg Universität Mainz, 55099 Mainz, Germany

e-mail: werth@uni-mainz.de

F. Schmidt-Kaler

Institut für Physik, Universität Mainz, Staudingerweg 7, 55128 Mainz, Germany

R. Blatt

Institut für Experimentalphysik, Universität Innsbruck, Technikerstrasse 25/4, 6020 Innsbruck, Austria following years a tremendous increase in applications, important technical developments such as linear traps, and the miniaturization of trapping chip devices. The invention of the frequency comb technique by Theodor Hänsch and Jen Hall in 2005 to determine even optical frequencies led to frequency standards in the optical and near UV domain with unprecedented accuracy. The formation of ion crystals and cooling into the quantum mechanical ground state of the ion's oscillation in the trap's harmonic potential opened the path for the new and exciting field of quantum information processing. The 2012 Nobel Prize in Physics awarded to D. Wineland (together with S. Haroche) "for ground-breaking experimental methods that enable measuring and manipulation of individual quantum systems" clearly recognizes the revolutionary development in singleparticle spectroscopy and manipulation during the last two decades, which became possible using Paul traps.

In August 2013, Wolfgang Paul would have celebrated his 100th birthday. In order to commemorate this date, we take this opportunity to edit a special volume dedicated to ion trap physics. Even though the invention of the Paul trap dates back to more than half a century, the contributions to this volume clearly hallmark the active ongoing research with Paul traps. The ever increasing number of applications has had and continues to have an enormous impact in many fields of physics, especially for atomic clocks, quantum optics and quantum information processing, for accurate mass measurements, the study of chemical reactions at the single-particle level, for cluster physics and for nuclear precision measurements as well as for ion-atom hybrid systems. Paul traps continue to be an excellent physics tool for decades to come. 